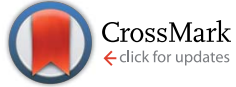

Cite this: Food Funct., 2014, 5, 1932

Received 14th January 2014 Accepted 19th May 2014

DOI: $10.1039 / c 4 f o 00029 c$

www.rsc.org/foodfunction

\section{High levels of Bifidobacteria are associated with increased levels of anthocyanin microbial metabolites: a randomized clinical trial $\dagger$}

\author{
María Boto-Ordóñez, ${ }^{a}$ Mireia Urpi-Sarda, ${ }^{a}$ María Isabel Queipo-Ortuño, ${ }^{\text {bc }}$ \\ Sara Tulipani, ${ }^{\text {bb }}$ Francisco J. Tinahones ${ }^{c d}$ and Cristina Andres-Lacueva*a
}

\begin{abstract}
The health benefits associated with the consumption of polyphenol-rich foods have been studied in depth, however, the full mechanism of action remains unknown. One of the proposed mechanisms is through microbiota interaction. In the present study, we aimed to explore the relationship between changes in fecal microbiota and changes in urinary phenolic metabolites after wine interventions. Nine participants followed a randomized, crossover, controlled interventional trial. After the washout period, they received red wine, dealcoholized red wine or gin for 20 days each. Polyphenol metabolites $(n>60)$ in urine were identified and quantified by UPLC-MS/MS and the microbial content of fecal samples was quantified by real-time quantitative PCR. Interventions with both red wine and dealcoholized red wine increased the fecal concentration of Bifidobacterium, Enterococcus and Eggerthella lenta, compared to gin intervention and baseline. When participants were categorized in tertiles of changes in fecal bacteria, those in the highest tertile of Bifidobacteria had higher urinary concentration changes in syringic acid, $p$ coumaric acid, 4-hydroxybenzoic acid and homovanillic acid (all anthocyanin metabolites) than those in tertile $1(P<0.05$, all). In addition, changes of Bifidobacteria correlated positively with changes of these metabolites ( $r=0.5-0.7, P<0.05$, all). Finally, the $68.5 \%$ changes in Bifidobacteria can be predicted by syringic acid and 4-hydroxybenzoic acid changes. This study confirms the important role of polyphenols as bacterial substrates and their modulatory capacity as an important field in the research of new products with prebiotic and probiotic characteristics for the food industry.
\end{abstract}

\section{Introduction}

In the nutrition research field the link between food consumption and human health has been explored and discussed in depth. From macronutrients ${ }^{1}$ to micronutrients ${ }^{2}$ and phytochemicals, ${ }^{3}$ the knowledge of their true role in the prevention of diseases has been limited to the high individual variability and understanding of the human metabolism. In this process, microbiota have been positioned as a key point due to their relation with some diseases such as obesity ${ }^{4}$ and metabolic syndrome $^{5}$ or in host defense. ${ }^{6}$ The connection between the diet and microbiota is described in two ways: (i) food may have a

${ }^{a}$ Biomarkers and Nutritional \& Food Metabolomics Research Group, Nutrition and Food Science Department, XaRTA, INSA, Pharmacy Faculty, University of Barcelona, Avenida Joan XXIII s/n, 08028 Barcelona, Spain.E-mail: candres@ub.edu

${ }^{b}$ Research Laboratory, IMABIS Foundation, Virgen de la Victoria Clinical Hospital, Málaga, Spain

${ }^{c}$ CIBER Fisiopatología de la Obesidad y la Nutrición, Instituto de Salud Carlos III, Madrid, Spain

${ }^{d}$ Servicio Endocrinología y Nutrición del Hospital Universitario Virgen de la Victoria, Málaga, Spain

$\dagger$ Electronic supplementary information (ESI) available. See DOI: $10.1039 / \mathrm{c} 4$ fo00029c prebiotic effect modulating the bacterial composition; ${ }^{7,8}$ (ii) and behaving as a metabolic entity, producing metabolites derived from food consumption. ${ }^{9}$ One of the diet components that have been associated with health benefits through microbiota interaction is polyphenols. ${ }^{\mathbf{1 0}}$ Most dietary polyphenols arrive intact at the colon and may interact with microbiota to be metabolized before being absorbed. ${ }^{11}$ This interaction could involve hydrolysis, demethylation, reduction, decarboxylation, dehydroxylation and isomerization of these compounds into more simple components ${ }^{\mathbf{1 2}}$ modulating absorption and biological activity. ${ }^{\mathbf{1 3}}$ Concurrently, polyphenols and metabolites formed in the intestine have the ability to promote and inhibit the growth of bacterial groups. ${ }^{9}$ In the case of red wine (RW), it has been described that the interaction of polyphenols with microbiota releases a wide range of microbial metabolites found in biofluids ${ }^{\mathbf{1 4 1 5}}$ and also increases the number of Enterococcus, Prevotella, Bacteroides, Bifidobacterium, Bacteroides uniformis, Eggerthella lenta, and Blautia coccoides-Eubacterium rectale groups along with the phyla Proteobacteria, Fusobacteria, Firmicutes and Bacteroidetes. ${ }^{7}$ However, the link between bacterial groups and polyphenolic metabolites after food consumption has just been proved in in vitro studies, where 
microbiota incubated with polyphenols release phenolic metabolites whose presence may modulate their growth. ${ }^{\mathbf{1 6 , 1 7}}$

For that reason, we embarked on a study to evaluate the association between changes in the bacterial number produced at the intestinal level and urinary changes in microbial phenolic acids in a randomized, crossover, controlled intervention study divided into three periods of 20 days each of RW, dealcoholized red wine (DRW) or gin consumption.

\section{Materials and methods}

\section{Study subjects and design}

The study was an open, randomized, crossover, controlled intervention trial ${ }^{7}$ that involved 9 adult men aged between 45 and 50. The study design was divided into 3 consecutive periods of 20 days each with an initial washout period (baseline) during which the participants did not consume any alcohol or red wine. This period was followed in a random order by 3 consecutive periods during which the participants drank DRW (272 $\mathrm{mL}$ per day), or RW (272 mL per day, containing $30 \mathrm{~g}$ ethanol), or gin (100 mL day ${ }^{-1}$, containing $30 \mathrm{~g}$ ethanol).

At baseline, and after each intervention period, participants provided fecal and $24 \mathrm{~h}$ urine samples, which were stored at $-80{ }^{\circ} \mathrm{C}$ until analysis. They were asked to maintain their dietary habits and pattern and lifestyle and to avoid alcoholic beverages during the whole study. No significant differences were observed in daily energy and dietary intake at the beginning of the study and after each intervention. ${ }^{7}$

Participants had not received treatment for diabetes, hypertension, or dyslipidemia, any antibiotic therapy, prebiotics, probiotics, symbiotics, or vitamin supplements or any other medical treatment influencing intestinal microbiota during the 3 months before the start of the study or during the study (including the washout period). They did not have any acute or chronic inflammatory diseases, infectious diseases, viral infections, or cancer, and had not had a previous cardiovascular event at study entry. The Ethics Committee of the Virgen de la Victoria Hospital approved the clinical protocol. All the participants gave written informed consent. This trial was registered at http://www.controlled-trials.com as ISRCTN88720134.

\section{Red wine, dealcoholized red wine and gin}

The RW and DRW used in this study were elaborated with the Merlot grape variety, from the Penedès appellation (Catalonia). No differences in the phenolic composition were found in the RW and DRW. ${ }^{7}$

\section{Chemicals and reagents}

Available phenolic acids and flavanols and $\beta$-glucuronidase/ sulfatase (from Helix pomatia) were purchased from SigmaAldrich (St. Louis, MO, USA), PhytoLab GmbH \& Co. KG (Vestenbergsgreuth, Germany), and Extrasynthèse (Genay, France) as previously described. ${ }^{\mathbf{1 4}}$ HPLC grade solvents were purchased from Scharlau Chemie, S.A. (Sentmenat, Spain) and Panreac Química, S.A.U. (Castellar del Vallès, Spain). Ultrapure water (Milli-Q) was obtained from a Millipore System (Bedford, MA, USA).

\section{Sample extraction}

Microbial-derived and conjugated metabolites present in urine were analyzed using solid-phase extraction (SPE) with an Oasis ${ }^{\circledR}$ MCX and HLB 96-well plates (Waters, Milford, Massachusetts), respectively, as previously described. ${ }^{\mathbf{1 4 , 1 8 , 1 9}}$ Briefly, urine samples $(1 \mathrm{~mL})$ were loaded onto the conditioned cartridge plate, washed and eluted with methanol or acidified methanol, respectively, and evaporated to dryness. Reconstitution of the residues was carried out with $100 \mu \mathrm{L}$ of taxifolin in the mobile phase.

\section{UPLC-MS/MS analysis}

Metabolites in urine were analyzed by UPLC-MS/MS equipped with a binary solvent manager and a refrigerated autosampler plate (Waters Acquity UPLC system, Milford, MA, USA), coupled to an AB Sciex API 3000 triple quadrupole mass spectrometer equipped with a turbo ion spray, ionizing in negative mode (PE Sciex). An Acquity UPLC BEH C18 column (Milford, MA, USA) $(1.7 \mu \mathrm{m}, 2.1 \mathrm{~mm} \times 5 \mathrm{~mm})$, using a prefilter, working at $40{ }^{\circ} \mathrm{C}$ and with an injection volume of $5 \mu \mathrm{L}$, was used as described before. ${ }^{14}$ Mobile phases used were: A (0.1\% formic acid) and B $\left(0.1 \%\right.$ formic acid in acetonitrile) at a flow rate of $500 \mu \mathrm{L} \mathrm{min}{ }^{-1}$ with the following proportions $(\mathrm{v} / \mathrm{v})$ of phase A $[\mathrm{t}(\mathrm{min}), \% \mathrm{~A}]$ : (0,92); (2.5,50); (2.6,0); (3,0); (3.1,92); (3.5,92). The MS/MS parameters used were as previously described..$^{\mathbf{1 4}}$ Phase II and microbial metabolites were quantified using the Multiple Reaction Monitoring (MRM) mode with a dwell time of $10 \mathrm{~ms}$. Calibration curves were constructed with available standards in synthetic urine and subjected to the same procedure as the samples. If a standard was not available, metabolites were quantified using the most similar compound standard curve and results were expressed as their equivalents. ${ }^{14}$ The metabolites analyzed for this study are shown in the ESI. $\dagger$ Quality parameters of the methodology were accomplished with accuracy, precision and recovery $<15 \% .{ }^{19}$

\section{DNA extraction from fecal samples and analysis of fecal microbiota by polymerase chain reaction (PCR)}

Extraction of DNA was from $200 \mathrm{mg}$ stools by using a QIAamp DNA Stool Mini Kit (Qiagen) and concentration and purity were estimated with a NanoDrop spectrophotometer (NanoDrop Technologies). For bacterial quantification to characterize the fecal microbiota, specific primers targeting different bacterial genera were used by PCR as previously described. ${ }^{7}$ Briefly, the LightCycler 2.0 PCR sequence detection system, by using the FastStart DNA Master SYBR Green Kit (Roche Diagnostics), was used for quantitative PCR experiments. Comparison among $C_{\mathrm{t}}$ values obtained from the standard curves with the LightCycler 4.0 software was carried out to calculate the bacterial concentration. Standard curves were created by using a serial 10 -fold dilution of DNA from pure cultures, corresponding to $10^{1}$ to $10^{10}$ copies per $\mathrm{g}$ feces. The data presented were the mean values of duplicate real-time qPCR analyses. 


\section{Statistical analysis}

Before the statistical analysis, a cube root transformation and a range scaling of phenolic data through the MetaboAnalyst Webbased platform was performed for normalization ${ }^{\mathbf{1 4 , 2 0}}$ and the bacterial copy numbers were converted into logarithm values. ${ }^{7}$ In this work, we only considered those bacteria whose levels differed between both wine intakes and gin/baseline periods: two bacterial genera (Bifidobacterium, Enterococcus) and one species (Eggerthella lenta). ${ }^{7}$ Changes of bacteria and phenolic acids after wine intervention were assessed by checking the difference compared to baseline. The procedure consisted in categorizing the participants based on tertiles of changes of bacterial genera or species. To study the differences of urinary metabolites through bacterial genera or specie tertiles, we used one-way analysis of variance (ANOVA) (IBM SPSS Statistics software program for Windows version 20 (Chicago, IL)). If changes of metabolites and bacteria presented a significant Spearman correlation, lineal regression stepwise analysis was performed in order to establish which of these metabolites were predictors of bacterial changes. Statistical significance was considered to be $P<0.05$.

\section{Results}

\section{Tertiles of bacterial group change after wine consumption}

In this study, we considered bacteria that showed significant modifications after both wine interventions compared to gin intervention and to baseline. ${ }^{7}$ No differences in the number of bacteria (means $\pm \mathrm{SD}, \log _{10}$ copies per $\mathrm{g}$ feces) were observed between DRW and RW: Bifidobacterium (9.93 \pm 1.85 and $9.88 \pm$ 1.78, respectively), Eggerthella lenta (9.84 \pm 1.65 and $9.97 \pm 1.77$, respectively) and Enterococcus (6.94 \pm 1.5 and $7.10 \pm 1.1$, respectively). ${ }^{7}$ Tertiles of differences were calculated between the bacterial number after wine interventions and baseline. The tertile distribution is presented in Fig. 1. The third tertile showed higher mean increases of Bifidobacterium (5.52 \pm 0.88 $\log _{10}$ copies per $\mathrm{g}$ feces), Enterococcus $\left(2.83 \pm 0.51 \log _{10}\right.$ copies per $\mathrm{g}$ feces) and Eggerthella lenta $\left(3.47 \pm 1.03 \log _{10}\right.$ copies per $\mathrm{g}$

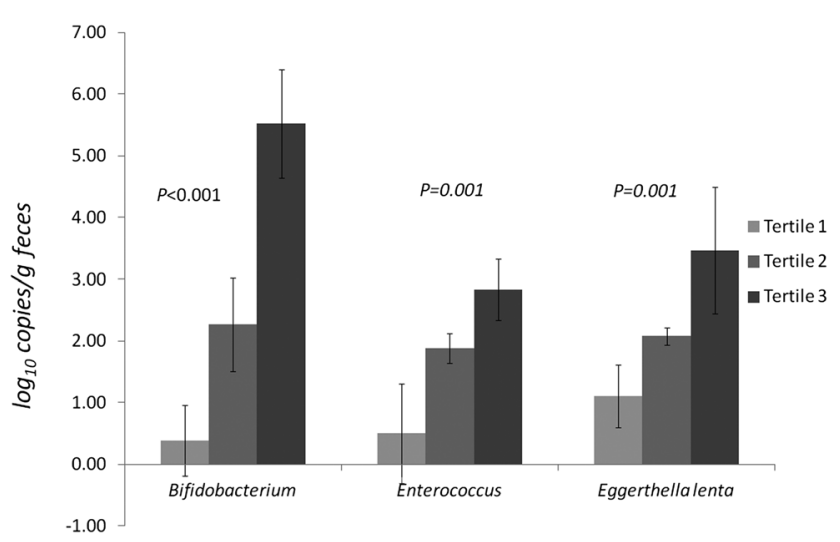

Fig. 1 Tertiles of bacterial differences of two genera (Bifidobacterium and Enterococcus) and one species (Eggerthella lenta) between wine interventions and baseline (mean \pm SD). feces) while the first tertile showed lower mean increases of Bifidobacterium $\left(0.38 \pm 0.57 \log _{10}\right.$ copies per $\mathrm{g}$ feces), Enterococcus ( $0.50 \pm 0.81 \log _{10}$ copies per $\mathrm{g}$ feces) and Eggerthella lenta $\left(1.10 \pm 0.51 \log _{10}\right.$ copies per $\mathrm{g}$ feces). The increases through tertiles of bacteria were significant $(P<0.001)$.

\section{Relationship among changes in bacterial population and urinary phenolic metabolites}

The tertiles of bacterial modifications were used to analyze phenolic metabolite changes after wine interventions (Table 1). From the lowest to the highest changes in Bifidobacteria tertiles, participants showed a higher excretion of four phenolic metabolites related to anthocyanin metabolism (Fig. 2): syringic, $p$-coumaric, 4-hydroxybenzoic and homovanillic acids. On the other hand, higher increases of Eggerthella lenta corresponded to lower excretion of hydroxycinnamates and syringic acid. In addition, higher increases of Enterococcus corresponded to lower excretion of 3,4-dihydroxyphenylacetic acid.

Additionally, correlation analysis indicated that only differences in Bifidobacteria were significantly correlated with differences in syringic $(r=0.537, P<0.022), p$-coumaric $(r=$ $0.621, P<0.006)$, 4-hydroxybenzoic $(r=0.677, P<0.002)$ and homovanillic acids $(r=0.507, P<0.032)$. Linear regression stepwise analysis evaluating Bifidobacteria changes included only two metabolites in the model $\left(R^{2}=0.685 ; P<0.001\right)$, syringic acid and 4-hydroxybenzoic acid (Table 2 ). This model explained $68.5 \%$ of the Bifidobacterium changes.

\section{Discussion}

The increased knowledge about the role of microbiota in human health and the possible modulation through food consumption is an interesting field for developing new products in the food industry such as probiotics and prebiotics. ${ }^{21}$ Food has demonstrated the capacity to modulate the growth of intestinal bacteria in several clinical trials ${ }^{8,22}$ and produce bioactive metabolites. ${ }^{23}$

One of the main studied bacteria to be affected by food intake is Bifidobacteria. Bifidobacterium is one of the predominant genera in the human intestine, and it is considered a health-promoting constituent of the microbiota. ${ }^{24}$ In this study, the unique metabolites correlated with Bifidobacterium were those derived from anthocyanin degradation: 4-hydroxybenzoic, syringic, $p$-coumaric and homovanillic acids. The concentration of anthocyanins in wine is high but lower than that of flavanols, which are the main wine polyphenols. ${ }^{15,25}$ Nevertheless, their dietetic distribution in Mediterranean diets is more limited than flavan-3-ols and their metabolites have been proposed as excellent markers of wine consumption. ${ }^{14}$ Anthocyanins were first supposed to have low bioavailability, ${ }^{26}$ but in the last few years, studies with isotopically labeled anthocyanins have demonstrated that anthocyanins reach the colon where they are transformed, releasing new metabolites that differ from the original compound. ${ }^{27}$

Microbial metabolism of anthocyanins at the colonic level involves reactions of breakage in the C-ring, resulting in 
Table 1 Changes in urinary phenolic metabolites after wine interventions (mean value \pm standard deviation) according to changes in bacterial population tertiles

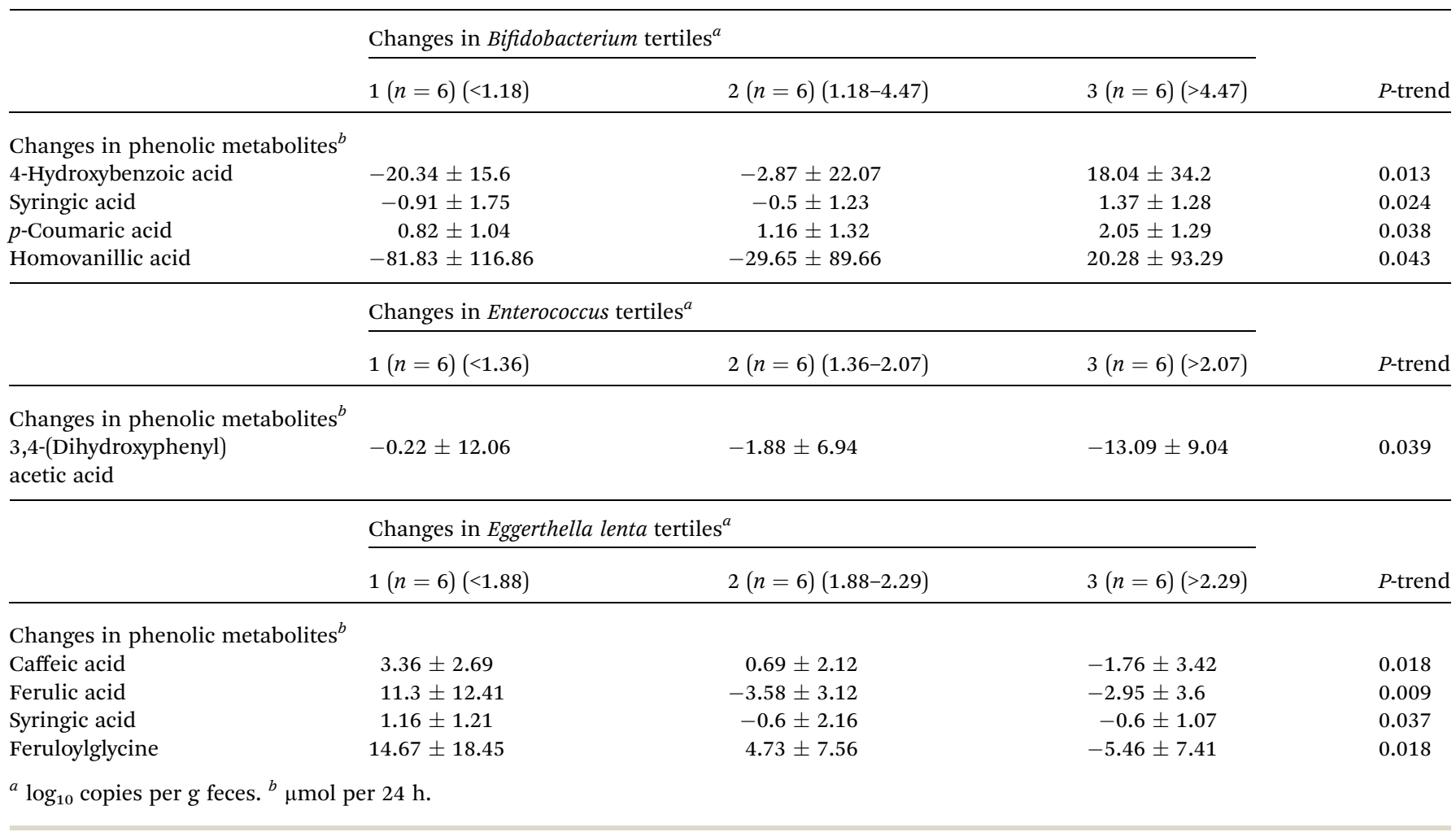

hydroxylated aromatic compounds derived from the A-ring, and release of the B-ring in numerous phenolic acids, depending on their hydroxylated pattern, ${ }^{12}$ as well as deglycosylation. ${ }^{28}$ Furthermore, Bifidobacterium enzymatic activity for polyphenols has not only been associated with ring fission, ${ }^{28}$ but also hydrolysis ${ }^{29}$ and glycosidase activity. ${ }^{28}$ Fig. 2 shows the principal origin of microbial metabolites derived from the anthocyanin structure associated with Bifidobacteria increase. One of these phenolic acids is 4-hydroxybenzoic acid, which has been proposed as a pelargonidin metabolite, ${ }^{30}$ and comes from microbial degradation of $p$-coumaric ${ }^{31}$ or could come from syringic acid demethylation, a reaction associated with certain intestinal bacteria. ${ }^{\mathbf{1 1}}$ Moreover, some studies have shown that the concentration of 4-hydroxybenzoic acid increased in plasma and urine after strawberry consumption by healthy volunteers ${ }^{32}$ and in the urine of rats fed with wine powder. ${ }^{33}$ Syringic acid may come from malvidin degradation described from Lactobacillus and Bifidobacterium. ${ }^{28,34}$ These two metabolites were the ones that entered the stepwise logistic regression, indicating that they were the strongest contributors to Bifidobacteria change after wine consumption. In the same study, $p$-coumaric acid was also formed when delphinidin and malvidin were incubated with these bacteria ${ }^{28}$ via hydrolysis of $p$-coumaroylacylated anthocyanins, which are abundant in red wine (Fig. 2). Homovanillic acid has also been described as coming from malvidin glycoside degradation via demethoxylation and was one of the main urinary metabolites after berry purée consumption by humans. ${ }^{35}$
It is difficult to establish whether these compounds are primary anthocyanin metabolites or are derived from other sources. Homovanillic acid could also be formed from ferulic acid, ${ }^{35}$ additionally $p$-coumaric acid could come from dehydroxylation of caffeic acid, and syringic acid from gallic acid. ${ }^{36}$ Moreover, some of these metabolites, such as gallic acid, are also present in the original wine composition..$^{15,25}$

Previous studies have already shown the role of anthocyanins in the bifidogenic effect as Guglielmetti et al. found after consumption of a wild blueberry drink by humans. ${ }^{37}$ Biological effects associated with these changes have already been described. Bifidobacterium has been associated with antiobesity effects $^{38}$ and cholesterol regulation. ${ }^{39}$ Metabolite 4-hydroxybenzoic could be responsible for the antioxidant properties of polyphenol consumption, inhibiting tyrosine nitration through the formation of 4-hydroxy-3-nitrobenzoic acid ${ }^{\mathbf{4 0}}$ Syringic acid has been proved to increase nitric oxide production ${ }^{41}$ and coumaric acid has inhibitory activity over angiotensin-converting enzymes. ${ }^{42}$ The biological activities attributed to the increase in metabolites could be responsible for benefits observed in blood pressure and improving the plasma lipid profile or inflammation in this study. ${ }^{22,43}$

The other bacterial species modified after red wine consumption was Eggerthella lenta, which is significantly abundant in intestinal microbiota. ${ }^{24}$ Significant inverse associations were found between changes in Eggerthella lenta tertiles and changes in hydroxycinnamic acid concentrations and between changes in Enterococcus tertiles and changes in 

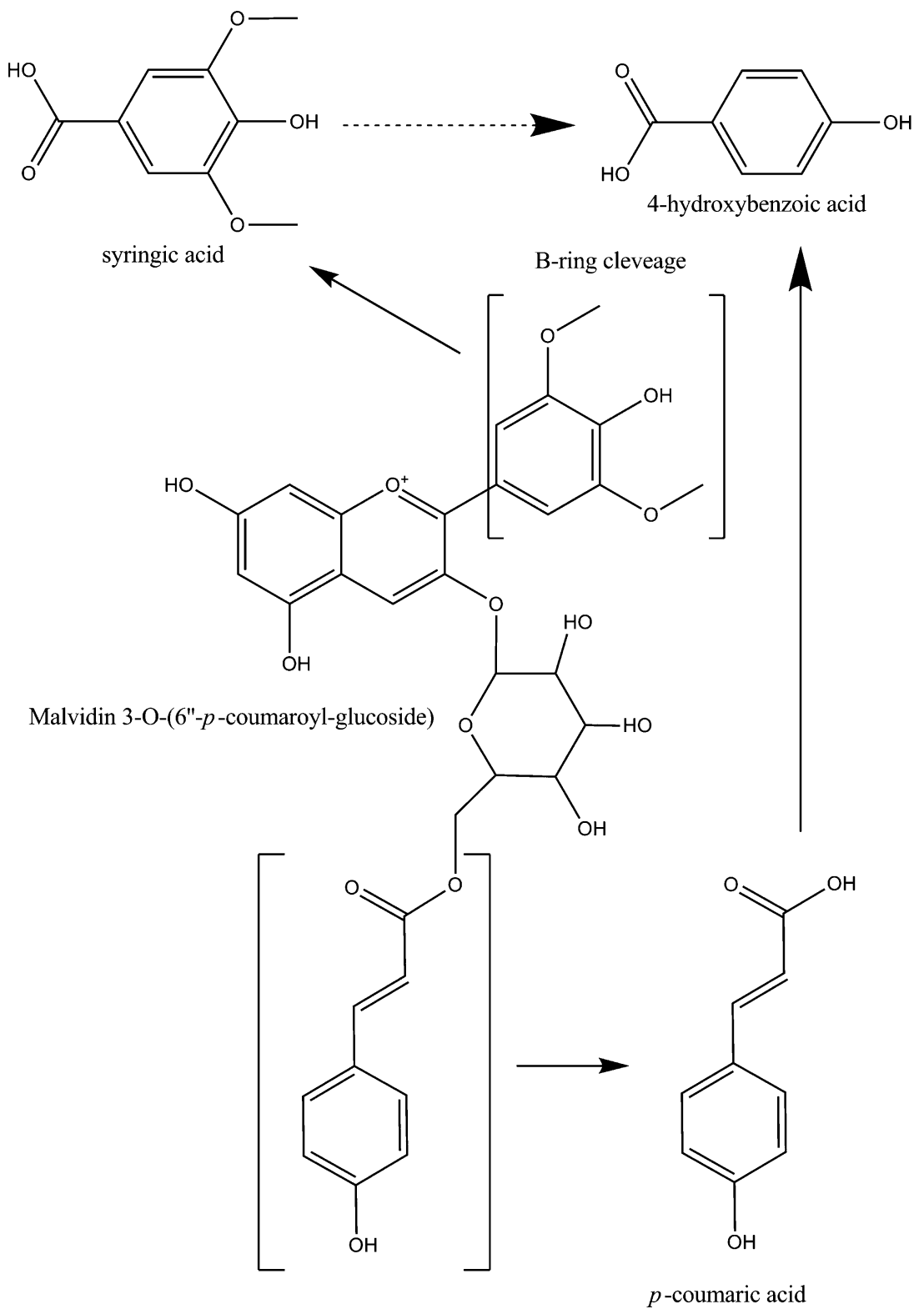

Fig. 2 Proposed metabolic route of anthocyanin degradation by Bifidobacteria.

Table 2 Stepwise linear regression model showing the best metabolite predictors of Bifidobacterium change

\begin{tabular}{lllr}
\hline & $B(\mathrm{SD})$ & $\beta$ & $P$ \\
\hline $\begin{array}{l}\text { Bifidobacterium } \\
\text { Intercept }\end{array}$ & $2.75(0.37)$ & & \\
$\begin{array}{l}\text { 4-Hydroxybenzoic } \\
\text { acid }\end{array}$ & $3.60(1.27)$ & 0.513 & 0.0013 \\
Syringic acid & $3.63(1.51)$ & 0.402 & 0.042 \\
& & &
\end{tabular}

3,4-dihydroxyphenylacetic acid concentration. This was probably due to the fact that both bacterial groups could be inhibited by phenolic compounds including gallic acid and resveratrol metabolites in in vitro studies. ${ }^{4-46}$ In addition, Enterococcus genus bacteria have been inhibited by cloudberry intake. ${ }^{47}$ Although we found significant inverse associations between Eggerthella lenta and Enterococcus tertiles and some phenolic acid concentrations, changes in Eggerthella lenta and Enterococcus cannot be predicted by phenolic acids changes.

One of the main limitations of this study was the lack of washout periods between interventions, however, no carryover effect was observed, and the absence was therefore unlikely to affect the results obtained. ${ }^{7}$ Moreover, the inclusion of washout periods between interventions would extend the study a further 6 weeks, creating difficulty to ensure compliance, so the subjects would be more inclined to withdraw from the study. ${ }^{7}$ In addition, the limitations of this study suggest the need for future next steps that potentially will be to increase the number of subjects. So, future studies would be designed to answer if 
changes in microbiota levels produced changes in phenolic acid concentration or inversely.

\section{Conclusion}

Bacterial changes after red wine consumption, with or without alcohol content have been associated with the excretion of phenolic metabolites. Specifically, Bifidobacteria increase correlates with increases in microbial metabolites derived from wine anthocyanins. Numerous in vitro studies have shown the ability of intestinal bacteria to metabolize polyphenols and release them to the medium. Those metabolites have been found in plasma, urine and tissues after food consumption. To our knowledge this is the first approach where colonic bacteria in feces and microbial metabolites present in biofluids are studied from the same volunteer in an in vivo study.

This study contributes with new data to understanding the role of phenolic compounds in the maintenance of intestinal health and opens the way to considering anthocyanins not only as new prebiotics, but also as being responsible for health benefits associated with the consumption of anthocyaninrich food.

\section{Acknowledgements}

This work has been supported by CICYT AGL2006-14228-C0302, AGL2009-13906-C02-01 and the Ingenio-CONSOLIDER program, FUN-C-FOOD (CSD2007-063) from the Spanish Ministerio de Economía y Competitividad (MINECO). M.B.-O. would like to thank the FPU predoctoral program from the Spanish Ministry of Education, Culture and Sport. M.U.-S. would like to thank the "Ramon y Cajal" program from the MINECO and the Fondo Social Europeo. M.I. Q.-O. would like to thank "Miguel Servet Type I" program (CP13/00065) from the Instituto de Salud Carlos III. S.T. would like to thank the "Juan de la Cierva" program from the MICINN. We thank Torres S.A. for providing the red wine and dealcoholized red wine used in the study, and Gin Xoriguer for providing the gin used in the study. The authors have declared no conflict of interest.

\section{References}

1 W. C. Willett, Science, 1994, 264, 532-537.

2 E. L. Doets, L. S. de Wit, R. A. Dhonukshe-Rutten, A. E. Cavelaars, M. M. Raats, L. Timotijevic, A. Brzozowska, T. M. Wijnhoven, M. Pavlovic, T. H. Totland, L. F. Andersen, J. Ruprich, L. T. Pijls, M. Ashwell, J. P. Lambert, P. van 't Veer and L. C. de Groot, Eur. J. Nutr., 2008, 47(suppl. 1), 17-40.

3 M. H. Traka and R. F. Mithen, Plant Cell, 2011, 23, 24832497.

4 T. E. Sweeney and J. M. Morton, JAMA Surg., 2013, 148, 563569.

5 P. Kovatcheva-Datchary and T. Arora, Best Pract. Res., Clin. Gastroenterol., 2013, 27, 59-72.

6 I. M. Carroll, D. W. Threadgill and D. S. Threadgill, Mamm. Genome, 2009, 20, 395-403.
7 M. I. Queipo-Ortuno, M. Boto-Ordonez, M. Murri, J. M. Gomez-Zumaquero, M. Clemente-Postigo, R. Estruch, F. Cardona Diaz, C. Andres-Lacueva and F. J. Tinahones, Am. J. Clin. Nutr., 2012, 95, 1323-1334.

8 X. Tzounis, A. Rodriguez-Mateos, J. Vulevic, G. R. Gibson, C. Kwik-Uribe and J. P. Spencer, Am. J. Clin. Nutr., 2011, 93, 62-72.

9 J. van Duynhoven, E. E. Vaughan, D. M. Jacobs, R. A. Kemperman, E. J. van Velzen, G. Gross, L. C. Roger, S. Possemiers, A. K. Smilde, J. Dore, J. A. Westerhuis and T. Van de Wiele, Proc. Natl. Acad. Sci. U. S. A., 2011, 108(suppl. 1), 4531-4538.

10 J. C. Espin, M. Larrosa, M. T. Garcia-Conesa and F. TomasBarberan, J. Evidence-Based Complementary Altern. Med., 2013, 2013, 270418.

11 M. V. Selma, J. C. Espin and F. A. Tomas-Barberan, J. Agric. Food Chem., 2009, 57, 6485-6501.

12 T. Requena, M. Monagas, M. A. Pozo-Bayón, P. J. MartínÁlvarez, B. Bartolomé, R. del Campo, M. Ávila, M. C. Martínez-Cuesta, C. Peláez and M. V. MorenoArribas, Trends Food Sci. Technol., 2010, 21, 332-344.

13 M. Monagas, M. Urpi-Sarda, F. Sanchez-Patan, R. Llorach, I. Garrido, C. Gomez-Cordoves, C. Andres-Lacueva and B. Bartolome, Food Funct., 2010, 1, 233-253.

14 M. Boto-Ordonez, M. Urpi-Sarda, M. I. Queipo-Ortuño, D. Corella, F. J. Tinahones, R. Estruch and C. AndresLacueva, J. Agric. Food Chem., 2013, 61, 9166-9175.

15 M. Boto-Ordonez, J. A. Rothwell, C. Andres-Lacueva, C. Manach, A. Scalbert and M. Urpi-Sarda, Mol. Nutr. Food Res., 2013, 58, 466-477.

16 F. Sanchez-Patan, C. Cueva, M. Monagas, G. E. Walton, G. R. Gibson, J. E. Quintanilla-Lopez, R. Lebron-Aguilar, P. J. Martin-Alvarez, M. V. Moreno-Arribas and B. Bartolome, J. Agric. Food Chem., 2012, 60, 2136-2147.

17 R. Kemperman, G. Gross, S. Mondot, S. Possemiers, M. Marzorati, T. Van de Wiele, J. Dore and E. E. Vaughan, Food Res. Int., 2013, 53, 659-669.

18 M. Urpi-Sarda, M. Monagas, N. Khan, R. Llorach, R. M. Lamuela-Raventos, O. Jauregui, R. Estruch, M. Izquierdo-Pulido and C. Andres-Lacueva, J. Chromatogr. A, 2009, 1216, 7258-7267.

19 M. Urpi-Sarda, M. Monagas, N. Khan, R. M. LamuelaRaventos, C. Santos-Buelga, E. Sacanella, M. Castell, J. Permanyer and C. Andres-Lacueva, Anal. Bioanal. Chem., 2009, 394, 1545-1556.

20 J. Xia, N. Psychogios, N. Young and D. S. Wishart, Nucleic Acids Res., 2009, 37, W652-W660.

21 X. He, M. L. Marco and C. M. Slupsky, J. Agric. Food Chem., 2013, 61, 9559-9574.

22 M. I. Queipo-Ortuno, L. M. Seoane, M. Murri, M. Pardo, J. M. Gomez-Zumaquero, F. Cardona, F. Casanueva and F. J. Tinahones, PLoS One, 2013, 8, e65465.

23 G. Williamson and M. N. Clifford, Br. J. Nutr., 2010, 104(suppl. 3), S48-S66.

24 M. Arumugam, J. Raes, E. Pelletier, D. Le Paslier, T. Yamada, D. R. Mende, G. R. Fernandes, J. Tap, T. Bruls, J. M. Batto, M. Bertalan, N. Borruel, F. Casellas, L. Fernandez, 
L. Gautier, T. Hansen, M. Hattori, T. Hayashi, M. Kleerebezem, K. Kurokawa, M. Leclerc, F. Levenez, C. Manichanh, H. B. Nielsen, T. Nielsen, N. Pons, J. Poulain, J. Qin, T. Sicheritz-Ponten, S. Tims, D. Torrents, E. Ugarte, E. G. Zoetendal, J. Wang, F. Guarner, O. Pedersen, W. M. de Vos, S. Brunak, J. Dore, M. Antolin, F. Artiguenave, H. M. Blottiere, M. Almeida, C. Brechot, C. Cara, C. Chervaux, A. Cultrone, C. Delorme, G. Denariaz, R. Dervyn, K. U. Foerstner, C. Friss, M. van de Guchte, E. Guedon, F. Haimet, W. Huber, J. van HylckamaVlieg, A. Jamet, C. Juste, G. Kaci, J. Knol, O. Lakhdari, S. Layec, K. Le Roux, E. Maguin, A. Merieux, R. Melo Minardi, C. M'Rini, J. Muller, R. Oozeer, J. Parkhill, P. Renault, M. Rescigno, N. Sanchez, S. Sunagawa, A. Torrejon, K. Turner, G. Vandemeulebrouck, E. Varela, Y. Winogradsky, G. Zeller, J. Weissenbach, S. D. Ehrlich and P. Bork, Nature, 2011, 473, 174-180.

25 V. Neveu, J. Perez-Jimenez, F. Vos, V. Crespy, L. du Chaffaut, L. Mennen, C. Knox, R. Eisner, J. Cruz, D. Wishart and A. Scalbert, Database, 2010, 2010, bap024.

26 A. Bub, B. Watzl, D. Heeb, G. Rechkemmer and K. Briviba, Eur. J. Nutr., 2001, 40, 113-120.

27 C. Czank, A. Cassidy, Q. Zhang, D. J. Morrison, T. Preston, P. A. Kroon, N. P. Botting and C. D. Kay, Am. J. Clin. Nutr., 2013, 97, 995-1003.

28 M. Ávila, M. Hidalgo, C. Sánchez-Moreno, C. Pelaez, T. Requena and S. D. Pascual-Teresa, Food Res. Int., 2009, 42, 1453-1461.

29 L. Roncaglia, A. Amaretti, S. Raimondi, A. Leonardi and M. Rossi, Appl. Microbiol. Biotechnol., 2011, 92, 159-168.

30 G. M. Woodward, P. W. Needs and C. D. Kay, Mol. Nutr. Food Res., 2011, 55, 378-386.

31 A. Sachan, S. Ghosh, S. K. Sen and A. Mitra, Appl. Microbiol. Biotechnol., 2006, 71, 720-727.

32 W. R. Russell, L. Scobbie, A. Labat and G. G. Duthie, Mol. Nutr. Food Res., 2009, 53(suppl. 1), S85-S91.

33 M. P. Gonthier, V. Cheynier, J. L. Donovan, C. Manach, C. Morand, I. Mila, C. Lapierre, C. Remesy and A. Scalbert, J. Nutr., 2003, 133, 461-467.
34 M. Hidalgo, M. J. Oruna-Concha, S. Kolida, G. E. Walton, S. Kallithraka, J. P. Spencer and S. de Pascual-Teresa, J. Agric. Food Chem., 2012, 60, 3882-3890.

35 T. Nurmi, J. Mursu, M. Heinonen, A. Nurmi, R. Hiltunen and S. Voutilainen, J. Agric. Food Chem., 2009, 57, 2274-2281.

36 A. Nurmi, T. Nurmi, J. Mursu, R. Hiltunen and S. Voutilainen, J. Agric. Food Chem., 2006, 54, 6916-6923.

37 S. Guglielmetti, D. Fracassetti, V. Taverniti, C. Del Bo, S. Vendrame, D. Klimis-Zacas, S. Arioli, P. Riso and M. Porrini, J. Agric. Food Chem., 2013, 61, 8134-8140.

38 H. M. An, S. Y. Park, K. Lee do, J. R. Kim, M. K. Cha, S. W. Lee, H. T. Lim, K. J. Kim and N. J. Ha, Lipids Health Dis., 2011, 10, 116.

39 D. I. Pereira and G. R. Gibson, Appl. Environ. Microbiol., 2002, 68, 4689-4693.

40 T. Tsuda, Y. Kato and T. Osawa, FEBS Lett., 2000, 484, 207210.

41 T. Simoncini, E. Lenzi, A. Zochling, S. Gopal, L. Goglia, E. Russo, K. Polak, E. Casarosa, A. Jungbauer, A. D. Genazzani and A. R. Genazzani, Maturitas, 2011, 70, 169-175.

42 M. Hidalgo, S. Martin-Santamaria, I. Recio, C. SanchezMoreno, B. de Pascual-Teresa, G. Rimbach and S. de Pascual-Teresa, Genes Nutr., 2012, 7, 295-306.

43 M. Clemente-Postigo, M. I. Queipo-Ortuno, M. BotoOrdonez, L. Coin-Araguez, M. M. Roca-Rodriguez, J. Delgado-Lista, F. Cardona, C. Andres-Lacueva and F. J. Tinahones, Am. J. Clin. Nutr., 2013, 97, 1053-1061.

44 C. M. Jung, T. M. Heinze, L. K. Schnackenberg, L. B. Mullis, S. A. Elkins, C. A. Elkins, R. S. Steele and J. B. Sutherland, FEMS Microbiol. Lett., 2009, 297, 266-273.

45 H. C. Lee, A. M. Jenner, C. S. Low and Y. K. Lee, Res. Microbiol., 2006, 157, 876-884.

46 G. Özkan, O. Sagdiç, N. Göktürk Baydar and Z. Kurumahmutoglu, J. Sci. Food Agric., 2004, 84, 1807-1811. 47 R. Puupponen-Pimia, L. Nohynek, C. Meier, M. Kahkonen, M. Heinonen, A. Hopia and K. M. Oksman-Caldentey, J. Appl. Microbiol., 2001, 90, 494-507. 